\title{
Red de Investigación de Retinopatía Diabética: Retinopatía diabética y edema macular diabético. Artículo de revisión
}

\section{Diabetic Retinopathy Clinical Research Network: Diabetic retinopathy and diabetic macular edema. Review article}

\author{
José L. Palomares-Ordóñez*, Sergio Rojas-Juárez, Jorge A. Sánchez-Ramos, Jazmín A. Salas-García, \\ Ingrid Pita-Ortíz, Angeles Y. Hernández, Carla R. Pérez-Montaño y Juan A. Ramírez-Estudillo \\ Departamento de Retina y Vítreo, Fundación Hospital Nuestra Señora de la Luz, IAP, Ciudad de México, México
}

\section{Resumen}

La Red de Investigación de Retinopatía Diabética (DRCR.net), fundada por el Instituto Nacional Ocular, se formó en 2002. Actualmente está conformada aproximadamente por 400 oftalmólogos de 115 centros clínicos de 37 estados de la Unión Americana. Desde entonces ha conducido múltiples estudios sobre diversos puntos de interés en torno a la retinopatía diabética y el edema macular diabético. A fecha de hoy, la DRCR.net tiene alrededor de 80 publicaciones, entre ensayos clínicos (nombrados con letras alfabéticas), estudios observacionales y diversos subanálisis derivados de los estudios principales. Los resultados obtenidos proveen a los especialistas en retina de evidencia con respecto a los protocolos de tratamiento clásicamente propuestos, además de estudiar nuevas pautas de tratamiento e información con respecto a otros puntos de importancia sobre las patologías estudiadas. La gran cantidad de información proporcionada por protocolo y los correspondientes subanálisis hacen de su comprensión una tarea difícil. En el presente trabajo se pretende resaltar y resumir los puntos importantes y conclusiones de cada uno de los protocolos ya publicados por el DRCR.net, y remarcar su importancia en la práctica clínica, lo que resultará en mejores decisiones para el tratamiento individualizado de los pacientes diabéticos.

Palabras clave: Ensayo clínico. Análisis. Retinopatía diabética. Edema macular diabético.

\begin{abstract}
The diabetic retinopathy clinical research network (DRCR.net), founded by the National Eye Institute, was formed in 2002. Currently it includes over 400 ophthalmologists in 115 clinical centers in 37 states of the United States. Since then, it has conducted several studies on various points of interest about diabetic retinopathy and diabetic macular edema. To date, the DRCR.net has published about 80 articles, including clinical trials (named with alphabetic letters), observational studies and various sub analyses derived from the primary studies. The results obtained provide retina specialists with substantial evidence regarding the treatment protocols classically proposed. Also, they evaluate new treatment guidelines and information concerning other important points about the disorders studied. The large amount of information provided by the protocols and the corresponding sub analyses make understanding them a difficult task. In this work, we intend to emphasize and
\end{abstract}

Correspondencia:

*José L. Palomares-Ordóñez

Ezequiel Montes, 135

Col. Tabacalera, Del. Cuauhtémoc

Fecha de recepción: 15-08-2018

Fecha de aceptación: 08-12-2018 DOI: 10.24875/RMO.M18000063
Disponible en internet: 01-03-2019 Rev Mex Oftalmol. 2019;93(2):84-97 www.rmo.com.mx cia CC BY-NC-ND 
summarize the essential points and conclusions of each of the protocols already published by the DRCR.net, highlighting their importance in clinical practice, which will result in better decisions for the individualized treatment of diabetic patients.

Key words: Clinical trial. Analysis. Diabetic retinopathy. Diabetic macular edema.

\section{Protocolo A}

El Early Treatment Diabetic Retinopathy Study (ETDRS) demostró que la fotocoagulación láser focal o en parrilla reducía al 50\% el riesgo de baja visual moderada (BVM) causada por el edema macular diabético (EMD) durante los siguientes 3 años a su aplicación. La efectividad del láser no se podía explicar en su totalidad, aunque se sugiere que el cierre de la fuga causada por los microaneurismas podría ser de los mecanismos más importantes, al menos en cierta parte. Posteriormente, algunos clínicos observaron una respuesta favorable tras la aplicación del láser focal 0 en parrilla a pesar de no impactar directamente sobre los microaneurismas y, así mismo, hubo observaciones de efectos desfavorables tras aplicaciones excesivas de tratamiento en áreas muy cercanas al centro macular, por lo que surgió la idea de generar un nuevo esquema de tratamiento diferente al propuesto por el ETDRS.

El protocolo A comparó dos técnicas de fotocoagulación láser para el tratamiento del EMD: la técnica modificada propuesta por el ETDRS (láser directo o en parrilla) (grupo 1) contra una técnica en parrilla macular leve (MMG) (grupo 2). La primera surge con la intención de reducir los efectos adversos observados por la proximidad al centro macular y la expansión de las quemaduras. La segunda, como una alternativa propuesta por el DRCR.net, que aplica quemaduras más leves, alejadas del centro macular y con espacios más amplios. Característicamente, sobre la retina aparentemente sana, no engrosada, sin aplicación directa sobre microaneurismas. Los esquemas de tratamiento se detallan en la Tabla 1.

Se aleatorizaron 263 pacientes (323 ojos) con EMD virgen a tratamiento a recibir una de las dos técnicas de fotocoagulación.

Se analizó la agudeza visual (AV), se realizó tomografía de coherencia óptica dominio tiempo (OCT-DT) mediante Stratus 3.0 y fotografía de fondo estándares del ETDRS basal y en cada visita, a los 3.5 meses, 8 meses y 12 meses, así como fluorangiografía (FAG) basal y al año. Se repitió el esquema de fotocoagulación en cada visita si el edema persistía.

El objetivo primario fue observar el cambio en diversas medidas de la OCT-DT a los 12 meses. Los cambios en la AV mejor corregida (AVMC) fueron considerados como objetivos secundarios.

Como resultado, a los 12 meses se observó que en los ojos con grosor macular central $(\mathrm{GMC})>250 \mu \mathrm{m}$ basal, el GMC tuvo una disminución media de $88 \mu \mathrm{m}$ en el grupo 1 contra $49 \mu \mathrm{m}$ en el grupo $2(p=0.02)$. El volumen macular disminuyó $0.8 \mathrm{~mm}^{3}$ y $0.4 \mathrm{~mm}^{3}$, respectivamente $(p=0.03)$. El cambio medio en la AVMC fue de +0 letras y -2 letras, respectivamente $(p=0.10)$.

Se concluyó una menor efectividad en la técnica MMG en comparación con la técnica modificada ETDRS'1. En un subanálisis se comparó el GMC con la AVMC antes y después del tratamiento, y se encontró una correlación modesta posterior al tratamiento con fotocoagulación láser. Se resalta que la toma de AV no puede ser sustituida a pesar de que la OCT-DT resulta una herramienta importante en la evaluación, así mismo demostró que los cambios en la OCT-DT, tanto a corto como largo plazo, no son predictores del cambio en la $\mathrm{AV}^{2}$.

Al hacer correlación entre OCT-DT y fotografías estereoscopias de fondo para evaluar el grosor retiniano, se observó de igual manera una débil relación con la AV, pero moderada correlación entre OCT-DT y fotografía de fondo en evaluar el GMC y una ligera superioridad en la reproducibilidad, así como una mayor sensibilidad con la OCT-DT en evidenciar cambios en el grosor retiniano ${ }^{3}$.

También se estudió si la extensión del EMD valorada por OCT-DT pretratamiento, explicaría la AV inicial y podría precedir el cambio en el GMC o la AV posterior al láser, pero no se obtuvo respuesta con el subanálisis ${ }^{4}$.

Al evaluar la asociación entre las características en la FAG con AV, OCT-DT y fotografías de fondo, se vio qué área de fuga basal se asocia con $\mathrm{AV}$ reducida y valores de grosor y volumen por OCT-DT aumentados basales, pero sin asociaciones a cambios a los 12 meses $^{5}$.

\section{Protocolo B}

Durante la década de los años noventa se iniciaron las investigaciones sobre el uso de diversos agentes para su uso intravítreo, los cuales pudieran tener efecto benéfico en diferentes patologías, dentro de ellas el 
Tabla 1. Comparación de los parámetros para fotocoagulación láser de la técnica macular directa o en parrilla modificada de la propuesta por ETDRS y la técnica en parrilla macular leve por DRCR.net

\begin{tabular}{|c|c|c|}
\hline $\begin{array}{l}\text { Características de la } \\
\text { quemadura }\end{array}$ & $\begin{array}{l}\text { Fotocoagulación focal/parrilla (técnica } \\
\text { modificada-ETDRS) }\end{array}$ & $\begin{array}{l}\text { Fotocoagulación en parrilla macular } \\
\text { leve (MMG-DRCR.net) }\end{array}$ \\
\hline Tratamiento directo & $\begin{array}{l}\text { Directamente tratar todos los microaneurismas } \\
\text { con fuga en el área de retina engrosada entre } \\
500 \text { a } 3,000 \text { micras del centro macular (pero no } \\
\text { dentro de las } 500 \text { micras alrededor del disco } \\
\text { óptico) }\end{array}$ & No aplica \\
\hline $\begin{array}{l}\text { Cambio del color del } \\
\text { microaneurisma con } \\
\text { tratamiento directo }\end{array}$ & $\begin{array}{l}\text { No requerido, pero al menos quemadura gris } \\
\text { moderada, que debe ser evidente debajo de } \\
\text { todos los microaneurismas }\end{array}$ & No aplica \\
\hline $\begin{array}{l}\text { Tamaño de la quemadura con } \\
\text { tratamiento directo }\end{array}$ & 50 micras & No aplica \\
\hline $\begin{array}{l}\text { Duración de la quemadura con } \\
\text { tratamiento directo }\end{array}$ & 0.05 a $0.1 \mathrm{~s}$ & No aplica \\
\hline Tratamiento en parrilla & $\begin{array}{l}\text { Aplicada a todas las áreas de fuga difusa o de no } \\
\text { perfusión dentro del área descrita para } \\
\text { tratamiento }\end{array}$ & $\begin{array}{l}\text { Aplicada a toda el área descrita para } \\
\text { tratamiento arriba (incluyendo retina no } \\
\text { engrosada) }\end{array}$ \\
\hline $\begin{array}{l}\text { Área considerada para } \\
\text { tratamiento en parrilla }\end{array}$ & $\begin{array}{l}500 \text { a } 3,000 \text { micras superior, nasal e inferior del } \\
\text { centro macular, } 500 \text { a } 3500 \text { micras temporales al } \\
\text { centro macular. Alejadas al menos } 500 \text { micras del } \\
\text { disco óptico }\end{array}$ & $\begin{array}{l}500 \text { a } 3,000 \text { micras superior, nasal e inferior del } \\
\text { centro macular, } 500 \text { a } 3,500 \text { micras temporales } \\
\text { al centro macular. Alejadas al menos } 500 \\
\text { micras del disco óptico }\end{array}$ \\
\hline $\begin{array}{l}\text { Tamaño de la quemadura con } \\
\text { tratamiento en parrilla }\end{array}$ & 50 micras & 50 micras \\
\hline $\begin{array}{l}\text { Duración de la quemadura con } \\
\text { tratamiento en parrilla }\end{array}$ & 0.05 a $0.1 \mathrm{~s}$ & 0.05 a $0.1 \mathrm{~s}$ \\
\hline $\begin{array}{l}\text { Intensidad de la quemadura } \\
\text { con tratamiento en parrilla }\end{array}$ & Apenas visible (gris claro) & Apenas visible (gris claro) \\
\hline $\begin{array}{l}\text { Separación de la quemadura } \\
\text { con tratamiento en parrilla }\end{array}$ & Separación de dos diámetros de quemadura & $\begin{array}{l}\text { Total de } 200 \text { a } 300 \text { quemaduras ( } 2 \text { a } 3 \text { diámetros } \\
\text { de quemadura de separación) }\end{array}$ \\
\hline $\begin{array}{l}\text { Longitud de onda del } \\
\text { tratamiento focal o en parrilla }\end{array}$ & Longitudes de onda verde a amarilla & Longitudes de onda verde a amarilla \\
\hline
\end{tabular}

EMD. En el año 2001 y 2002 se publicaron los primeros reportes de casos aislados sobre el uso de triamcinolona intravitrea para el tratamiento del EMD, que sugirió cierta efectividad. Su uso se popularizó según encuestas de la Asociación Americana de Especialistas en Retina. Puesto que no existía un mayor nivel de evidencia sobre su seguridad y efectividad, se consideró importante realizar un ensayo clínico aleatorizado para evaluarla.

El estudio consistió en comparar la triamcinolona con el entonces estándar de tratamiento propuesto por el ETDRS para EMD, la fotocoagulación láser focal o en parrilla.

Se aleatorizó a los pacientes con EMD con involucración del centro de la mácula $(n=840)$ en 3 grupos: 1) triamcinolona $1 \mathrm{mg}$ intravítreo, 2) triamcinolona $4 \mathrm{mg}$ intravítreo o c) fotocoagulación focal/parrilla. Los pacientes fueron tratados al inicio del estudio y retratados en caso necesario en cada visita (con un intervalo de 4 meses). Los grupos de triamcinolona podían recibir láser de rescate en caso de cumplir con criterios de fallo.

Aquellos pacientes con disminución visual de 15 letras o más en dos visitas consecutivas separadas por 4 meses (BVM) podían recibir tratamiento alternativo a criterio del investigador (los pacientes en el grupo de láser podían recibir triamcinolona y los de los grupos de triamcinolona podía aplicarse láser). Los resultados a 4 meses demostraron mayor ganancia visual en el grupo de triamcinolona $4 \mathrm{mg}$ respecto a los otros dos; sin embargo, al año de seguimiento no hubo diferencia significativa entre los tres grupos. 
A los 2 y 3 años de seguimiento $(n=306)$ hubo una mayor ganancia de AV en el grupo de láser comparado con los dos grupos de triamcinolona. Esta diferencia no se consideró atribuible al desarrollo de catarata, ya que los resultados fueron consistentes al analizar a los pacientes pseudofáquicos, además que la disminución en el GMC fue similar en los tres grupos. Este estudio demostró que el láser focal/parrilla tiene mayor beneficio a largo plazo en comparación con la triamcinolona intravítrea en pacientes con EMD, además de presentar menor número de efectos adversos (aumento de la presión intraocular y progresión de la catarata) ${ }^{6,7}$.

\section{Protocolo C}

Algunos estudios evidenciaron variaciones en el grosor retiniano por OCT a lo largo del día en algunos pacientes con EMD: era mayor durante la mañana y menor en las últimas horas del día. Debido a que la OCT se ha convertido en la herramienta estándar para la evaluación del EMD, se consideró importante estudiar la variación diurna para una interpretación más precisa.

En este estudio ( $n=156)$, se realizó la medición del grosor retiniano mediante OCT-DT a las siguientes horas del día: $8,10,12,14$ y $16 \mathrm{~h}$. El promedio del grosor del subcampo central a las $8 \mathrm{~h}$ fue de $360 \mu \mathrm{m}$, con una disminución del $6 \%(13 \mu \mathrm{m})$ a las $16 \mathrm{~h}$. En 5 ojos (3\%) hubo una disminución de más del $25 \%$ del grosor retiniano. El cambio en el grosor retiniano fue significativamente mayor en ojos que tenían mayores grosores retinianos a las $8 \mathrm{~h}$. En el 10\% de los ojos hubo una ganancia de más de 10 letras de la cartilla ETDRS y un empeoramiento de 10 letras en el $6 \%$ de los ojos examinados. De acuerdo con los resultados de este estudio, se considera que, aunque puede existir una disminución progresiva del grosor retiniano a lo largo del día en pacientes con EMD, estos cambios son pequeños y ocasionales, además de tener una implicación clínica poco relevante, por lo que no debe influir en los horarios de medición de los ensayos clínicos ${ }^{8}$.

\section{Protocolo D}

El vítreo ha sido implicado, al menos en parte, como causa de edema macular (EM) por diversos mecanismos fisiológicos y mecánicos. Así mismo, se observó que la separación entre el vítreo y la superficie retiniana resultaba en una subsecuente reducción del grosor retiniano en pacientes con EMD, ya fuera mediante cirugía o mediante un desprendimiento espontáneo.
Por lo que, el DRCR.net realizó un estudio prospectivo y observacional $(n=87)$ para evaluar el resultado visual y anatómico (GMC con OCT-DT) posterior a vitrectomía sin cirugía concomitante de catarata en pacientes con EMD asociado a tracción vitreomacular (TVM). Adicionalmente a la vitrectomía, se realizó pelado de la membrana epirretiniana (MER) en el $61 \%$ de los casos, limitorrexis en el 54\%, panfotocoagulación retiniana (PFC) en el 40\%, así como inyección de triamcinolona al final del procedimiento en el 64\%. La AVMC basal media fue de 20/100 y el GMC basal de $491 \mu \mathrm{m}$. A los 6 meses, hubo una reducción media del GMC de $160 \mu \mathrm{m}$ y el $68 \%$ de los pacientes tuvo reducción de al menos el $50 \%$ del grosor. El $38 \%$ de los pacientes presentaron ganancia de $\geq 10$ letras, aunque el $22 \%$ presentó una pérdida de $\geq 10$ letras. Después de los 6 meses no se encontraron cambios significativos.

Estos resultados sugieren beneficioso considerar la vitrectomía para el manejo del EMD en ojos con BVM de aquellos pacientes que se evidencia asociación con TVM, puesto que la mayoría de los ojos presentaron reducción del GMC y entre el 28 y el $49 \%$ de los ojos presentaron mejoría visual significativa. Vale mencionar que entre el 13 y el $31 \%$ de los ojos documentaron empeoramiento visual significativo ${ }^{9}$.

Al analizar los factores asociados en aquellos ojos que presentaron mayor ganancia de AV, se observó que esta ocurrió en ojos con peores AVMC iniciales y en aquellos a quienes se les removió una MER, por lo que se sugiere que la remoción de la misma puede ser beneficiosa. La presencia de anomalías en la interfaz vitreorretiniana se asocia a mayor reducción del GMC, pero sin diferencias significativas en la AVMC final ${ }^{10}$.

\section{Protocolo E}

Las inyecciones de corticoesteroides peribulbares se utilizaron para el tratamiento del EM secundario a uveítis, con resultados favorables, por lo que se planteó su eficacia en el EMD. Se pensaba que la vía peribulbar tenía menos efectos adversos que la vía intravítrea, lo que justificaría usarla específicamente en pacientes con EMD que cursaran con buena visión, en quienes los riesgos de inyecciones intravítreas no justificaban su uso. Por ello, se realizó un estudio piloto $(n=129)$ para evaluar los efectos que tienen estos medicamentos a nivel subtenoniano anterior y posterior, con o sin fotocoagulación coadyuvante, en pacientes con EMD y buena AV ( $\geq 20 / 40$ ). Los pacientes se aleatorizaron en 5 grupos: 1) fotocoagulación focal, 2) inyección de $20 \mathrm{mg}$ de triamcinolona subtenoniana anterior, 
3) inyección de $20 \mathrm{mg}$ de triamcinolona subtenoniana anterior seguida por fotocoagulación focal después de 4 semanas, 4) inyección de $40 \mathrm{mg}$ de triamcinolona subtenoniana posterior y 5) inyección de $40 \mathrm{mg}$ de triamcinolona subtenoniana posterior seguida por fotocoagulación focal después de 4 semanas. Los resultados a las 34 semanas de seguimiento no demostraron una diferencia significativa en cuanto al grosor retiniano o AV entre los 5 grupos, además, se reportaron efectos adversos atribuibles a las inyecciones, como aumento de la presión intraocular y ptosis palpebral. En conclusión, las inyecciones de triamcinolona peribulbar no demostraron tener ningún beneficio significativo en pacientes con EMD y buena $\mathrm{AV}^{11}$.

\section{Protocolo F}

Los estudios DRS y ETDRS demostraron que la PFC reduce el riesgo de baja visual grave $(<5 / 200$ en 2 visitas consecutivas con un intervalo de 4 meses), por lo que se convirtió en la terapia estándar para la retinopatía diabética proliferativa (RDP). Pero esta no resulta inocua, ya que se han reportado varias complicaciones ( $p$. ej., aparición o empeoramiento de EM) y no hay un número estándar de sesiones para realizarla.

El objetivo fue comparar los efectos de la PFC sobre el EMD aplicada en una sola sesión (grupo 1) contra la dividida en 4 sesiones (grupo 2 ) en pacientes con RDP temprana o retinopatía no proliferativa grave con AV relativamente buena $(>20 / 32)$ y $\mathrm{GMC}<300 \mu \mathrm{m}$ por OCT-DT.

En el 18\% se determinó EM preexistente. Durante el seguimiento, el grosor retiniano tuvo tendencia a aumentar ligeramente respecto a los niveles basales, pero el GMC fue ligeramente mayor en el grupo 1 al

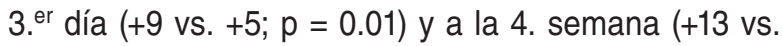
$+5 ; p=0.003$ ), y a la semana 34 , la diferencia se invirtió, y el grosor fue ligeramente mayor en el grupo 2 (+14 vs. +22; $p=0.06)$. Los resultados en AV fueron paralelos a los del GMC. A las 34 semanas, la AV fue ligeramente peor en el grupo 2 ( 0 vs. -2 letras; $p=0.006$ ) comparada con la basal. A pesar de los cambios reportados en la OCT-DT, solo un ojo en cada grupo requirió láser focal/parrilla.

Lgual que en otros estudios reportados, los resultados no mostraron diferencias significativas del efecto del tratamiento en la AV. No se identificaron efectos adversos en ningún grupo. Como conclusión, la PFC puede darse de forma segura en una sola sesión; sin embargo, este estudio no arroja suficiente evidencia que demuestre superioridad al compararla con la que se divide en mayor número de sesiones ${ }^{12}$.

\section{Protocolo G}

El EM es la principal causa de BVM en pacientes diabéticos. El diagnóstico es clínico y se complementa con la OCT macular. Está establecida la indicación de tratar el EMD que sea clínicamente significativo, pero se desconoce el valor de identificar el EMD subclínico. El EMD subclínico con involucración central se definió como el engrosamiento macular por OCT-DT de $\geq 225$ a $\leq 299 \mu \mathrm{m}$, pero que no se ve en la exploración clínica.

En un primer estudio realizado en 2008, el objetivo fue evaluar por OCT-DT el GMC en diabéticos sin retinopatía diabética $(\mathrm{RD})$ o con $\mathrm{RD}$ no proliferativa (RDNP) leve (solo microaneurismas) y sin engrosamiento macular central $(<225 \mu \mathrm{m})$ y con AV mejor a 20/32 ( $n=97$ ), y compararlo con los valores normativos de población sin enfermedad retiniana. El GMC promedio fue de $201 \pm 22 \mu \mathrm{m}$, y fue ligeramente mayor en los hombres. Ningún otro factor adicional, aparte del género, se encontró asociado a mayor grosor central macular. Concluyendo que el GMC, en este grupo de pacientes, no difiere de los valores normativos de población sin diabetes y que hay que tomar en cuenta la diferencia de género para la evaluación del grosor ${ }^{13}$.

En un estudio observacional en 2012 ( $n=43$ ), se determinó la tasa de progresión del EMD subclínico a EMD clínicamente significativo (incremento de $50 \mu \mathrm{m}$ del GMC o grosor $\geq 300 \mu \mathrm{m}$ ) o que requiriera tratamiento en un periodo de 2 años. A 1 año, 16/43 ojos cumplieron criterios de EM clínicamente significativo. La probabilidad de tener incremento en el GMC a 1 año fue del $27 \%$ y a 2 años del $38 \%$. Se demostró que el EM subclínico es poco frecuente (4.8\%), pero que tiene un porcentaje mayor (1/4 a 1/2 a 2 años) de progresión a EM clínicamente significativo que el reportado por el ETDRS (25\% a 3 años). Se sugiere la evaluación de este grupo de pacientes debido a su alto riesgo de progresión ${ }^{14}$.

\section{Protocolo H}

El bevacizumab es un anticuerpo monoclonal humanizado que inhibe todas las isoformas del factor de crecimiento vascular endotelial A (VEGF-A). Se ha utilizado ampliamente de manera off-label para el tratamiento del EMD.

Este es el primer estudio de fase 2 en evaluar el efecto del bevacizumab en el EMD. Se aleatorizaron 
121 pacientes a 5 grupos: 1) láser focal basal, 2) bevacizumab $1.25 \mathrm{mg}$ basal y a las 6 semanas, 3) bevacizumab $2.5 \mathrm{mg}$ basal y a las 6 semanas, 4) bevacizumab $1.25 \mathrm{mg}$ basal y reinyección simulada (sham) a las 6 semanas, o 5) bevacizumab $1.25 \mathrm{mg}$ basal y a las 6 semanas más láser focal a las 3 semanas. En la primera fase (12 semanas) se evaluó la eficacia y en la segunda fase se evaluó la seguridad hasta un periodo de 70 semanas. El objetivo primario fue el GMC por OCT-DT y la AVMC a las 24 semanas. Los grupos 2 y 3 tuvieron la mayor reducción del GMC a las 3 semanas y mejoría de 1 línea de AVMC a las 12 semanas, comparado con el grupo de láser solo, sin diferencia entre los grupos 2 y 3 . La combinación de láser con bevacizumab no mostró beneficios a corto plazo, pero tampoco efectos adversos. En cuanto a efectos adversos, se presentó un caso de endoftalmitis, dos casos de infarto agudo al miocardio y un evento de falla cardíaca congestiva. Las conclusiones fueron: el bevacizumab reduce más rápidamente el $\mathrm{GMC}$, el láser lo hace más lentamente, pero después de 3 semanas se equiparan; no hay diferencia a corto plazo entre ambas dosis en la reducción del GMC, y el efecto del bevacizumab tiene un pico inicial que se va estacionando, o incluso disminuye, a las 3 y 6 semanas, lo que sugiere que 6 semanas es mucho tiempo para una segunda dosis. Este estudio fue la base para los siguientes estudios de fase 3 realizados con bevacizumab ${ }^{15}$.

\section{Protocolo I}

Los beneficios del láser focal o en parrilla para el tratamiento del EMD demostrados por el ETDRS en 1985 fueron comprobados por el DRCR.net (protocolo B), donde también se mantuvo como el estándar de tratamiento frente a la triamcinolona, después de excluir al grupo de pacientes fáquicos que desarrollaron opacidad del cristalino. No obstante, en aquellos pacientes con $\mathrm{AV} \leq 20 / 40$, aproximadamente el $20 \%$ de aquellos tratados con láser focal o en parrilla presentaron un empeoramiento de $\geq 2$ líneas. Esto explica la necesidad de nuevas terapias que pudieran tener un mayor beneficio, como los anticuerpos monoclonales, ya fuera en monoterapia o como terapia coadyuvante al láser.

El protocolo I es un ensayo clínico, multicéntrico, en el que se aleatorizaron 854 ojos de estudio de 691 participantes con diagnóstico de EMD con involucración central. El principal objetivo del estudio consistió en evaluar el efecto de ranibizumab o triamcinolona en combinación con láser, comparado con monoterapia de láser, para el tratamiento del EMD ${ }^{16}$.

Se aleatorizaron en 4 grupos: 1) inyección intravítrea simulada más láser focal o en parrilla pronto (7-10 días) $(\mathrm{n}=293)$, 2) ranibizumab $0.5 \mathrm{mg}$ más láser pronto $(\mathrm{n}=187), 3)$ ranibizumab $0.5 \mathrm{mg}$ más láser diferido (>24 semanas) $(n=188), 04)$ triamcinolona 4 mg más láser pronto $(n=186)$.

El protocolo de tratamiento para la fotocoagulación láser fue administrado de acuerdo a la técnica modificada del protocolo original propuesto por el ETDRS, previamente descrito y utilizado en protocolos previos del DRCR.net; mientras que el protocolo de tratamiento con los agentes intravítreos se realizó de la siguiente manera: a partir del inicio del tratamiento hasta la semana 16, se aplicaron inyecciones cada 4 semanas sin importar la AV o el grosor macular; de la semana 16 a 20 se mantuvo la aplicación mensual, excepto cuando se presentaban "criterios de éxito" (AV > 20/20, GMC $<250 \mu \mathrm{m}$ ); de la semana 24 a 48, se categorizaron los pacientes con "criterios de éxito" y se difería el tratamiento, aquellos con mejoría (AV > 5 letras o mejoría en $<10 \%$ del GMC) continuaron mensualmente, aquellos sin criterios de mejoría se les asignó tratamiento a discreción del médico y, finalmente, aquellos con falla (AV peor a 10 letras que el basal, GMC > 250 $\mu \mathrm{m}$ y sin mostrar mejoría 13 semanas después de haber completado el tratamiento con láser) se les asignó un tratamiento «alterno» a discreción del médico; a partir de la semana 48 , se realizaron visitas cada 4 meses según el caso, si había sido diferido el tratamiento en visita previa, se extendía el seguimiento al doble de tiempo de la visita previa, con un máximo de intervalo de hasta 16 semanas.

Los resultados en el 1. ${ }^{\text {er }}$ año evidenciaron superioridad de ranibizumab, ya fuera con láser pronto $(+9 \pm 11)$ o diferido (+9 \pm 12$)$, en mejorar la AV cuando se compara con terapia combinada de triamcinolona más láser pronto $(+4 \pm 13)$ o con monoterapia de láser $(+3 \pm 13)$. La reducción en la media del GMC por OCT-DT fue similar para ranibizumab o triamcinolona. Así mismo, en pacientes pseudofáquicos $(n=273)$, la mejoría visual fue comparable para ambas terapias ${ }^{16,17}$.

A los 3 años de seguimiento se mantuvo la superioridad en los grupos de ranibizumab con láser pronto 0 diferido, y presentaron una diferencia en la AV media de +2.9 letras en el grupo de láser diferido en comparación con el de láser pronto. Igualmente, el grupo de láser diferido mostró un mayor porcentaje de pacientes con mejoría de $>10$ letras $(56 \%)$ en comparación con el grupo de láser pronto $(42 \%)^{18}$. 
Los resultados a 5 años de seguimiento mantuvieron proporciones similares a las diferencias iniciales, ranibizumab con láser pronto (+7.2) o diferido $(+9.8)$, con una diferencia media de -2.6 letras $(p=0.009)$. Los sujetos del grupo de tratamiento de ranibizumab más láser pronto 0 diferido requirieron una media de 8 a 9 inyecciones durante el $1 .^{\text {er }}$ año, 3 a 4 durante el $2 .^{\circ}$ año y 1 a 2 durante el $3 .^{\text {er }}$ año, respectivamente. Para el $4 .^{\circ}$ y $5 .^{\circ}$ años, la media del número de inyecciones fue de 0 para ambos grupos.

Así mismo, aquellos ojos asignados en los grupos de tratamiento de ranibizumab presentaron una menor tasa de progresión en aquellos pacientes con RDNP grave hacia RDP (8 vs. $42 \%$ ) aun cuando el esquema de inyecciones no se aplicó mensualmente después de la semana 12, que aquellos otros asignados al grupo de monoterapia con láser temprano. Por lo que fue demostrado, además, un efecto beneficioso de ranibizumab para el tratamiento de la neovascularización, lo que sugiere no requerir regímenes mensuales indefinidos para mantener estos efectos benéficos ${ }^{19}$.

\section{Protocolo J}

El empeoramiento del EMD preexistente posterior a PFC es un reconocido efecto adverso, aunque poco frecuente. El VEGF tiene un papel en el desarrollo y exacerbación del EMD, por lo que los esteroides y fármacos anti-VEGF podrían reducir la exacerbación del EMD preexistente en pacientes con RDP y RDNP grave.

Se aleatorizaron 345 ojos con $A V \geq 20 / 320$ y EMD tratados con láser focal/parrilla y con RD en tratamiento con PFC en 3 grupos: 1) inyección simulada basal y a las 4 semanas, 2) $0.5 \mathrm{mg}$ de ranibizumab basal y a las 4 semanas, y 3) triamcinolona basal e inyección simulada a las 4 semanas, para evaluar su efecto a corto plazo sobre el EMD. A las 14 semanas, el cambio medio en $\mathrm{AV}$ fue de $-4 \pm 14,+1 \pm 11$ y $+2 \pm 11$ en cada grupo, respectivamente, con mayor proporción de ojos con mejoría (> 10 letras) y menor proporción con empeoramiento (> 10 letras) en los grupos 2 y 3, respectivamente. Los cambios del grosor retiniano fueron paralelos a los de la AV. Evaluaciones subsecuentes a las 34 y 56 semanas solo tuvieron como objetivo evaluar la seguridad a largo plazo, aunque se observó que las diferencias en AV y GMC no se mantuvieron hasta las 56 semanas. Concluyendo que la adición de ranibizumab o triamcinolona al láser focal más PFC se asocia con una mayor AV y mayor disminución del GMC a las 14 semanas, pero no se puede determinar el beneficio a largo plazo con este estudio ${ }^{20}$.

\section{Protocolo K}

Los criterios de retratamiento mediante láser focal/ parrilla son un aspecto importante, y hasta cierto punto existía información limitada respecto a los resultados obtenidos tras una sola sesión de láser. El ETDRS y el DRCR.net retrataron tras cumplir criterios (persistencia de EM clínicamente significativo, identificación de una o más lesiones tratables que pudieran ser causa de baja visual) y con intervalos de separación de 4 meses.

El objetivo de este estudio consistió en determinar si la reducción del GMC asociada al láser focal o en parrilla aplicado en una sola sesión, continuaría aun tras diferir el tratamiento en pacientes con EMD con involucración central después de la semana 16. Se trató con fotocoagulación láser focal o en parrilla a 122 ojos con EM clínicamente significativo de acuerdo al esquema del ETDRS modificado. A partir de la semana 16, los pacientes se siguieron cada 8 semanas y se difirió el láser si presentaban mejoría de $>5$ letras en la cartilla ETDRS o disminución del GMC > 10\% con respecto al basal.

A la semana 16 , el $47 \%$ mostró mejoría de la AV. De estos, el $48 \%$ (26 ojos) tuvo $\geq 250 \mu \mathrm{m}$ de GMC, y fueron evaluados hasta la semana 32 . Solo $11 / 26$ ojos presentaron disminución del GMC de $>10 \%$ entre la semana 16 a la 32, sin necesidad de retratamiento.

Demostrando que, entre el 23 y el $63 \%$ de ojos que tuvieron mejoría pero no resolución del EMD a las 16 semanas después de la aplicación de láser, continuará mejorando sin necesidad de tratamiento hasta las 32 semanas. Evidencia útil para valorar esquema de retratamiento ${ }^{21}$.

\section{Protocolo L}

El ETDRS, además de aportar evidencia sólida referente a la RD y al EMD, estableció un método estandarizado para la valoración de la refracción óptica manual y consecuente AVMC (objetivos de investigación importantes). No obstante, la metodología presenta ciertas dificultades, dentro de las que se pueden destacar la necesidad del personal entrenado, tiempo prolongado y costos significativos. Una alternativa a esto, facilitada por el avance tecnológico, pudiera ser la autorrefracción.

El estudio incluyó de forma prospectiva 878 ojos de 456 participantes de 26 centros. El principal objetivo fue comparar el resultado de la AV obtenido después de realizar refracción automatizada con diferentes 
autorrefractores contra la refracción manual RM hecha de acuerdo al protocolo DRCR.net en pacientes con baja visual secundaria a EMD con amplio rango de AV (equivalente Snellen mejor a 20/400). El puntaje de AV fue determinado con la prueba de AV electrónica del ETDRS. A todos los participantes se les realizó la prueba de AV en tres ocasiones: una medición usando refracción obtenida mediante autorrefracción y dos mediciones adicionales usando refracción manual.

El equivalente esférico obtenido fue similar para ambas técnicas, con una media de diferencia de 0.00 con un rango de -1.75 a 1.13 dioptrías. Pero la AV media fue ligeramente mejor con las refracciones manuales.

La variabilidad obtenida entre ambas técnicas (refracción atomatizada contra refracción manual) fue considerablemente mayor que entre las dos mediciones manuales $(p<0.001)$ y fue altamente dependiente del modelo de autorrefractor. La menor variabilidad dentro de los diferentes equipos utilizados para la refracción automatizada fue la obtenida con las series Topcon 8000.

Los instrumentos para autorrefracción usados en este estudio no fueron capaces de sustituir la refracción manual, principalmente para ensayos clínicos por la alta variabilidad ${ }^{22}$.

\section{Protocolo M}

A pesar de que se ha establecido mediante diversos estudios que el control glucémico intensivo puede reducir la frecuencia y la progresión de complicaciones microvasculares en individuos con diabetes mellitus (DM), es claramente difícil de conseguir, especialmente a largo plazo. Algunos autores señalan obstáculos que impiden el control glucémico óptimo, resaltando la falta de consciencia sobre el potencial daño al órgano blan$\mathrm{co}$, aspectos culturales, así como limitaciones financieras, pobre acceso, depresión, falta de perseverancia y otros $^{23-26}$.

El DRCR.net realizó un estudio que se llevó a cabo en 42 centros de atención. El objetivo fue determinar si la combinación de evaluaciones de riesgos personalizadas y educación sobre la diabetes en una consulta de oftalmología puede mejorar el control de la glucemia en personas con diabetes, según lo evaluado por la hemoglobina glucosilada HbA1c con punto de corte primario a 1 año, extendido a 2 años.

Se aleatorizaron pacientes diabéticos a dos grupos: el que recibieron intervención y el de pacientes controles con atención estándar. Así mismo, cada grupo se subdividió en aquellos con seguimiento más frecuente que anual y aquellos con seguimiento anual. Los pacientes del grupo intervenido recibieron evaluación y educación personalizada que consistió en: a) punto de corte de la HbA1c, b) información sobre de tensión arterial y la gravedad de la retinopatía diabética (RD), c) riesgo personal para empeoramiento de la RD basado en el HbA1c y el tipo de diabetes, d) un reporte personalizado del riesgo de daño renal y retinopatía basado en la $\mathrm{HbA1c}$, e) una gráfica con los reportes previos y recientes de la $\mathrm{HbA1c}$, f) instrucciones para señalar los riesgos personales encontrados y g) material suplementario para el manejo de la diabetes. A los pacientes del grupo control se les requirió completar una visita mínima anual sin recibir mayor educación que la usual de la visita oftalmológica habitual.

El reporte medio de la $\mathrm{HbA1c}$ en los pacientes de seguimiento más frecuente que anual fue de $8.3 \%$ en el grupo control $(n=502)$ y $8.6 \%$ en el grupo de intervención $(n=488)$, con un cambio al año de $-0.1 \%$ y $-0.3 \%$, respectivamente, mientras que en los pacientes de seguimiento anual fue de $8.3 \%$ para el grupo control $(n=368)$ y de $8.4 \%$ para el grupo de intervención ( $n=388$ ), con un cambio de $0.0 \%$ y $-0.1 \%$, respectivamente. Como conclusión, se corrobora la dificultad de realizar un cambio en el comportamiento personal de los pacientes, aun con una educación adecuada sobre la patología y sus posibles complicaciones, igualmente sin presentar diferencia entre el seguimiento anual 0 de manera más frecuente ${ }^{27}$.

\section{Protocolo N}

La hemorragia vítrea secundaria a RDP se considera una de las principales indicaciones de vitrectomía en EE.UU., llevada a cabo cuando la hemorragia impide la adecuada aplicación de PFC. A pesar de los avances tecnológicos en cirugía de retina, las complicaciones no se pueden ignorar por completo. Algunas series de casos no controlados sugieren que los antiangiogénicos intravítreos causan un aclaramiento de la hemorragia vítrea a corto plazo como para poder aplicar PFC sin requerir vitrectomía.

Este estudio evaluó el efecto del ranibizumab intravítreo contra solución salina intravítrea para determinar si esto facilita el aclaramiento de la hemorragia vítrea y así evitar la vitrectomía. Se inyectó basal, y a las 4 y 8 semanas. A las 16 semanas, que fue el primer punto de corte, la probabilidad de vitrectomía fue del $12 \%$ para el grupo de ranibizumab y del $17 \%$ para el de solución salina balanceada $(p=0.37)$. La principal causa de vitrectomía entre las semanas 8 y 12 fue el 
no aclaramiento y, en una baja proporción, la progresión a Desprendimiento de Retina Traccional (DRT). También se observó una mejoría más acentuada en la $\mathrm{AV}$, mayor posibilidad acumulada de completar la PFC y menor tasa de recurrencia de la hemorragia vítrea, lo que sugiere un efecto biológico a corto plazo del ranibizumab ${ }^{28}$.

Posteriormente, en el análisis a 1 año, la diferencia en $\mathrm{AV}$ vista previamente no persistió y se mantuvo la ausencia de diferencias clínicas relevantes entre ambos grupos, con una ligera mayor posibilidad de completar la PFC en el grupo tratado con ranibizumab ${ }^{29}$.

\section{Protocolo 0}

Es importante para la práctica clínica, tanto como en el ámbito de la investigación, conocer con precisión, cuál es el GMC normal en ojos de individuos con DM pero sin EMD y diferenciarlo de aquellos que sí cursa con EMD, observados mediante OCT.

En un estudio previo se observó que pacientes con $\mathrm{DM}$ sin $\mathrm{RD}$, o mínima, así como aquellos con RD sin EMD, tienen grosores similares a pacientes sin DM y con retinas aparentemente normales. Todo ello determinado mediante la OCT-DT (OCT-3, Stratus).

Se realizó un primer estudio que evaluó el grosor macular de personas con diagnóstico de DM, sin RD o con RD mínima usando OCT de dominio espectral (OCT-DE, Spectralis, Heidelberg Engineering, Inc.).

Se realizaron las mediciones comparativas entre el Spectralis y Stratus de los 9 subcampos del ETDRS, además de hacer un comparativo con una base de datos normativa previamente reportada por el DRCR.net.

La media $(n=122)$ del GMC fue de $270 \pm 24 \mu \mathrm{m}$, con el punto central en rango de $227 \pm 25 \mu \mathrm{m}$, y el volumen medio fue de $8.4 \pm 0.4 \mathrm{~mm}^{3}$. Característicamente, los hombres presentaron mayores grosores y mayores volúmenes. En contraparte, el Stratus reportó una media del GMC de $199 \pm 24 \mu \mathrm{m}(71 \mu \mathrm{m}$ menor que el Spectralis) y un volumen medio de 6.7 $\mathrm{mm}^{3} \pm 0.4 \mathrm{~mm}^{3}$ (1.7 $\mathrm{mm}^{3}$ menor). Los resultados encontrados fueron comparables con datos obtenidos de individuos sanos sin DM.

Este estudio reportó la media de grosor encontrado en cada una de las 9 áreas del campo macular del ETDRS. Se proponen grosores $\geq 320 \mu \mathrm{m}$ en hombres $y \geq 305 \mu \mathrm{m}$ en mujeres como grosores específicos de género para determinar EM con involucración central de origen diabético ${ }^{30}$.

Se evaluó la reproducibilidad de los grosores retinianos de imágenes de OCT-DT, así como de OCT-DE, se realizaron ecuaciones para convertir las mediciones obtenidas mediante el Stratus y determinar su equivalente con el DE. Se determinó que existe una mejor reproducibilidad de las mediciones con el Spectralis cuando se compara con el Cirrus o Stratus ${ }^{31}$. Y al evaluar la reproducibilidad entre el GMC de Stratus contra aquellas obtenidas del Optovue RTVue, se concluyó que la variabilidad del RTVue es similar al Stratus o Cirrus ${ }^{32}$.

El análisis proporcionado por la red es útil para evaluar y comparar los resultados entre los ensayos y para el desarrollo de puntos de corte específicos de los diferentes SD-OCT disponibles para los ensayos clínicos posteriores.

\section{Protocolo P}

Aun cuando la cirugía de catarata se realiza en pacientes diabéticos de forma rutinaria, se cuenta con datos limitados sobre la AV y el comportamiento del GMC después de dicha cirugía en pacientes con EMD.

Este fue un estudio piloto, observacional, que tuvo como objetivo principal valorar la AV y el comportamiento del EM después de la cirugía de catarata en ojos de pacientes previamente diagnosticados con EMD con involucración central. Se incluyeron 63 pacientes con diagnóstico de EMD en al menos 1 ojo (GMC > $250 \mu \mathrm{m}$ mediante OCT-DT, $>310 \mu \mathrm{m}$ mediante OCT-DE). El 35\% $(n=21)$ de los pacientes no recibió tratamiento para el edema macular durante el estudio. El $43 \%(n=26)$ recibió tratamiento previo a la cirugía (láser focal/parrilla, triamcinolona intravítrea dentro de los 4 meses previos a la cirugía, antiangiogénico intravítreo dentro de los 2 meses previos a la cirugía). El $7 \%(n=4)$ recibió tratamiento intraoperatorio y el $42 \%$ $(n=25)$ recibió tratamiento posquirúrgico. De todos los pacientes que recibieron tratamiento, el $69 \%(n=27)$ recibió antiangiogénico intravítreo.

Al finalizar el seguimiento, en la semana 16 , se encontró un cambio en la media de la AV de +12 letras (IC 95\%: +8 a +16), se observó una mejoría de al menos 4 líneas en el 32\% (IC 95\%: 20-45\%) de los ojos, con un empeoramiento de la visión de al menos 2 líneas en un 10\% (IC 95\%: 4-21\%). De forma secundaria, se observó un cambio en la media del GMC por OCT de $-11 \mu \mathrm{m}$ (IC 95\%: -51a $28 \mu \mathrm{m})$ a las 16 semanas.

Este estudio cuenta con la deficiencia del bajo número de ojos estudiados en el lapso de tiempo establecido, asociado a un tratamiento heterogéneo para el EMD; sin embargo, con los resultados obtenidos, se estima que al menos la mitad de los ojos operados que tuvieron diagnóstico previo de EMD pueden no 
presentar mejoría visual. Debido al bajo poder de evidencia, no es posible asumir conclusiones definitivas en este estudio ${ }^{33}$.

\section{Protocolo Q}

Es ampliamente aceptado el hecho que la DM aumenta la probabilidad del desarrollo de catarata y podría aumentar el riesgo de mala visión después de la cirugía. Por otro lado, se ha reportado la cirugía de catarata como un factor de riesgo para la incidencia de EMD o para la progresión de la RD.

Este estudio tuvo como objetivo determinar la incidencia de EM con involucración central posterior a la cirugía de catarata en pacientes con RD sin engrosamiento macular central preexistente. Se aleatorizaron 293 pacientes en 4 grupos según el estudio de la OCT: 1) sin EMD, definido como grosor macular central menor a la media normal en todos los subcampos; 2) EMD «posible» en el campo central, definido como grosor entre la media y +2 desviaciones estándar de lo normal; 3) EMD «posible» no central, definido como grosor de al menos 1 campo no central entre la media y +2 desviaciones estándar, y 4) EMD «definitivo» no central, definido como al menos un campo no central de al menos +2 desviaciones estándar.

Se evaluaron los resultados 16 semanas después de la cirugía. Se inició con 329 participantes; sin embargo, después de ajustar los criterios de eliminación y el seguimiento de 16 semanas, se analizaron los datos de 261 ojos. Ningún ojo sin historia de EMD desarrolló EM con involucración central. Diez de los 97 (10\%; IC 95\%: $5-18 \%$ ) ojos sin involucración central previo a la cirugía desarrollaron EMD central, 18 de los 147 (12\%; IC 95\%: 7-19\%) ojos con «posible» involucración central desarrollaron EMD central. La tasa de desarrollo de EMD con involucración central a las 16 semanas de la cirugía de catarata difirió según la historia de tratamiento previo para EMD $(p<0.001)$. De los pacientes que habían sido tratados previamente para la EMD, el 21\% (IC 95\%: 14-30\%) desarrolló EM central, mientras que el 4\% (IC 95\%: 2- 9\%) de los que no tenían historia de tratamiento previo desarrolló EM central. En cuanto a la RD, el $82 \%$ no presentó cambios, mientras que el $10 \%$ progresó. También se encontró que aquellos ojos con mejor AV y RD menos grave al inicio del estudio presentaron menor incidencia de EMD central.

En conclusión, se demuestra que existe una mayor posibilidad de desarrollar EMD con involucración central después de una cirugía de catarata en pacientes con presencia de EM no central basal, así como con historia previa de EMD con involucración central previamente tratado ${ }^{34}$.

\section{Protocolo R}

El EMD es la causa más común de BVM en pacientes con RD. Se han relacionado diversas citocinas y otros factores proinflamatorios elevados en pacientes con diagnóstico de EMD. Lo que apoya a que esta podría ser responsable, al menos en parte, de la fisiopatología de la enfermedad. Los antiinflamatorios no esteroideos se han utilizado para la inflamación posquirúrgica, el nepafenaco tópico se usa frecuentemente para el tratamiento del edema macular quístico posquirúrgico.

Este estudio tuvo como principal objetivo evaluar la función del nepafenaco $0.1 \%$ tópico en ojos con EMD no central, definido como engrosamiento dentro de las $3.000 \mu \mathrm{m}$ del centro, sin involucración del centro macular.

Se aleatorizaron los pacientes en 2 grupos $(n=125)$. Grupo $1(n=61)$ : nepafenaco 3 veces al día. Grupo 2 $(n=64)$ : placebo. El seguimiento inicial fue de $12 \mathrm{me}-$ ses y no se permitió el tratamiento para EMD a menos que se observara involucración central.

El volumen medio inicial por TD-OCT fue de $7.8 \mathrm{~mm}^{3}$ y la media del grosor central fue de $223 \mu \mathrm{m}$. El cambio a los 12 meses fue de $-0.03 \mathrm{~mm}^{3}$ en el grupo $1 \mathrm{y}$ de $-0.02 \mathrm{~mm}^{3}$ en el grupo 2, sin diferencias al analizarse después de corregir el estado del cristalino y la HbA1c. En el grupo 1, 7 ojos (11\%) desarrollaron EMD central, mientras que en el grupo 2 lo desarrollaron 9 ojos (14\%). De los cuales, 5 ojos del grupo 1 y 3 ojos del grupo 2 requirieron tratamiento.

El cambio promedio en la AV fue de 0.09 letras y de -0.15 letras, respectivamente, con una diferencia de 0.2 letras entre los grupos. $(p=0.82)$. Entre los efectos secundarios se presentó un caso de lisis corneal en un ojo del grupo tratado con nepafenaco, el cual presentaba historia de ojo seco grave.

En conclusión, no se encontró beneficio en el uso tópico de nepafenaco durante 12 meses en cuanto al grosor retiniano mediante OCT ni en la AV, así mismo, el nepafenaco parece ser bien tolerado, sin mostrar diferencias en los efectos adversos en comparación con el grupo control ${ }^{35}$.

\section{Protocolo S}

La PFC ha sido el tratamiento estándar para la RDP desde lo reportado por el DRS y el ETDRS. Actualmente, 
con el advenimiento de la terapia antiangiogénica intravítrea, se han propuesto nuevos esquemas alternativos a la PFC, basados en el efecto antiangiogénico de estos fármacos.

EL protocolo $S$ evaluó la no inferioridad del ranibizumab de $0.5 \mathrm{mg}$ (Lucentis, Genetech) frente a la PFC para el tratamiento de la RDP con o sin EMD en pacientes vírgenes a PFC. Se aleatorizó a los participantes ( $n=305,294$ ojos) en 2 grupos. El grupo 1 recibió PFC, completada en 1 a 3 visitas, y el grupo 2 recibió ranibizumab basal y cada 4 semanas si cumplían los criterios de retratamiento, y podían recibir láser de rescate en caso de necesitarlo. En caso de presentar EMD, el grupo 1 podía recibir tratamiento antiangiogénico. En caso de hemorragia vítrea o desprendimiento de retina traccional, se indicó vitrectomía.

Como resultado principal se vio mejoría de +2.8 letras de la AVMC en el grupo 1 frente $a+0.2$ en el grupo $2(p<0.001)$ a los 2 años de seguimiento. En otros hallazgos, los pacientes del grupo 1 presentaron mayor pérdida de campo visual. La vitrectomía se realizó en el $15 \%$ de los pacientes del grupo 1 y en el $4 \%$ de los del grupo 2. Mayor número de pacientes en grupo 1 desarrolló EMD (28 vs. $9 \%$ ) y el $53 \%$ de pacientes del grupo 1 también recibieron ranibizumab y solo el $6 \%$ del grupo 2 recibió PFC.

A los 2 años no hubo diferencia estadística en cuanto a actividad o regresión de la neovascularización entre ambos grupos. No hubo diferencias en las tasas de desprendimiento de retina, glaucoma neovascular ni hemorragia vítrea en ambos grupos. La tasa de vitrectomía fue significativamente más alta en el grupo de PFC (15\%) que en el grupo de ranibizumab $(4 \%)^{36}$.

En un análisis sobre costo-beneficio en cuanto a la monoterapia con ranibizumab o PFC para RDP, se observó que era más adecuado iniciar monoterapia con PFC para pacientes con RDP pero sin EMD asociado, y ranibizumab para aquellos con EMD en el momento de la detección ${ }^{37}$.

En los resultados a 5 años $(n=184)$, el número promedio de inyecciones en el grupo 1 fue de 7.9 y en el grupo 2, de 19.2. El cambio medio en AV fue de +10.5 vs. +14.3 letras $(p=0.68)$, y la AVMC final media en ambos grupos fue de 20/25, que resultó consistente con los datos reflejados a los 2 años. A pesar de que a los 2 años el grupo 1 tuvo mayor pérdida de campo visual, la disminución del campo visual periférico progresó en ambos grupos durante los siguientes 5 años de seguimiento, tanto en el campo visual Humphrey 30-2 como en el 60-4. El cambio medio en el puntaje total acumulativo fue de -572 (635) dB vs. -330 (645) dB, y la diferencia en ambos grupos no fue estadísticamente significativa $(p=0.4)$. Más de la mitad de los ojos del grupo 1 recibió tratamiento con ranibizumab para $\mathrm{EMD}^{38}$.

Una pregunta importante que responde este protocolo es si el tratamiento con ranibizumab conlleva un riesgo de desarrollar complicaciones serias en ausencia de PFC después a 5 años de seguimiento. Pocos ojos en ambos grupos desarrollaron glaucoma neovascular o neovascularización en el iris, y la tasa de incidencia fue similar. La tasa global de desprendimiento de retina (en su mayoría traccional) fue más alta en el grupo 1 (15 vs. 6\%). Al menos la mitad de los ojos en ambos grupos, desarrolló algún grado de hemovítreo, pero solo el $42 \%$ en el grupo 1 y el $22 \%$ en el grupo 2 requirieron vitrectomía ${ }^{38}$.

En conclusión, el protocolo demuestra que ranibizumab no es inferior a PFC en cuanto a AVMC final, por lo que ambas terapias son adecuadas en el tratamiento de la RDP, principalmente para aquellos con EMD coexistente. Al seleccionar la terapia, siempre es importante tener presentes factores individuales del paciente, como la posibilidad o no de un seguimiento estrecho, costos y estado de control metabólico.

\section{Protocolo T}

Entre la gran variedad de antiangiogénicos disponibles, el aflibercept, el bevacizumab y el ranibizumab son los más frecuentemente usados, los tres han mostrado un beneficio importante y seguridad para el tratamiento del EMD.

En este protocolo se compararon los tres agentes. El total de pacientes $(n=660)$ se aleatorizó en 3 grupos: 1) aflibercept $2.0 \mathrm{mg}$, 2) bevacizumab $1.25 \mathrm{mg} \mathrm{o}$ 3) ranibizumab $0.3 \mathrm{mg}$. Se aplicó una dosis basal y después cada 4 semanas, excepto si cumplía con criterios de respuesta durante el primer año, y cada 4 a16 semanas el segundo año. EL objetivo principal fue evaluar el cambio en AVMC.

Durante el primer año, la mejoría en la AV fue de +13.3 con aflibercept, 9.7 con bevacizumab y 11.2 con ranibizumab, presentando diferencia estadística pero no representando significado clínico. Al hacer el análisis por rangos de visión, en el grupo de AV 20/32 a 20/40 no hubo diferencia estadística $(p>0.5)$, pero si la AV inicial era de 20/50 o peor, el aflibercept resultó superior a los otros dos fármacos (aflibercept vs. bevacizumab [ $p<0.001$ ], afliberceptvs vs. ranibizumab [ $p=0.003]$ ) y sin diferencia entre ranibizumab y bevacizumab ${ }^{39}$. 
En el análisis a 2 años, los resultados fueron similares. Los pacientes con mejor AVMC inicial consiguieron mejoría de forma similar con los tres diferentes fármacos. $Y$ aquellos con AVMC inicial de 20/50 o peor, aflibercept tuvo la mayor ganancia de AVMC; sin embargo, la diferencia fue menor que en el primer año y esta no fue estadísticamente significativa al compararla con ranibizumab, pero sí con bevacizumab. El número de inyecciones durante el $2 .^{\circ}$ año fue menor que el primero.

Se puede concluir que con cualquiera de los 3 antiangiogénicos, hay mejoría de AV, además de que los pacientes con peores AV basales se podrían ver beneficiados de tratamiento inicial con aflibercept ${ }^{40}$.

\section{Protocolo U}

Se ha demostrado el beneficio de los antiangiogénicos para la mejoría visual y la disminución del GMC en ojos con EMD. Sin embargo, al menos entre un 32 a $66 \%$ de los ojos tratados con antiangiogénico mensual durante 6 meses presentan edema persistente asociado a baja visual.

En este estudio se comparó el tratamiento con ranibizumab como monoterapia frente a la adición de implante de dexametasona en pacientes con EMD persistente (con EMD tratados con al menos 3 dosis de carga de algún antiangiogénico [bevacizumab, ranibizumab o aflibercept] dentro de las 20 semanas previas).

Se aleatorizaron ( $n=116)$ en 2 grupos: 1 ) ranibizumab 0.3 mg más implante de dexametasona $700 \mu \mathrm{g} \circ 2$ ) implante placebo más ranibizumab $0.3 \mathrm{mg}$. Se evaluaron cada 4 semanas durante 24 semanas y se revaluaba la necesidad de retratamiento.

La mejoría de AV fue de +2.7 letras en el grupo 1 y +3 letras en el grupo 2 (IC 95\%: -3.6 a 2.5; $p=0.73$ ). La mejoría de $\geq 10$ letras ocurrió en el $22 \%$ del grupo 1 y en el $14 \%$ del grupo 2. La disminución del GMC fue de $-110 \mu \mathrm{m}$ en el grupo 1 contra - $62 \mu \mathrm{m}$ en el grupo 2. En el grupo 1, el $29 \%$ desarrolló hipertensión intraocular en comparación con $<1 \%$ en el grupo $2(p<0.001)$. El total de inyecciones en las 24 semanas fue de 5.6 en el grupo 1 y 5.7 en el grupo 2.

En conclusión, el agregar el tratamiento con dexametasona a la terapia antiangiogénica no demostró una mejoría visual adicional, mientras que sí se determinó una disminución adicional del grosor macular central en comparación con el grupo tratado con monoterapia. Como se ha visto en estudios previamente realizados, el implante de liberación prolongada de dexametasona predispone a un aumento de la presión intraocular, por lo que su uso debe vigilarse estrechamente ${ }^{41}$.

\section{Protocolos actualmente en desarrollo}

\section{Protocolo V}

Protocolo cuyo objetivo será evaluar el tratamiento para EMD en ojos con muy buena visión. Incluirá ojos con EMD por OCT-DE, AV $\geq 20 / 25$. Los pacientes serán aleatorizados para recibir tratamiento con antiangiogénico (aflibercept y ziv-aflibercept) inmediato o láser pronto con antiangiogénico diferido para evaluar la proporción de ojos con pérdida $\geq$ a 5 letras en 2 años.

\section{Protocolo W}

Consiste en administrar antiangiogénico intravítreo (aflibercept) como terapia preventiva de características de alto riesgo de pérdida visual.

Tiene como objetivo primario evaluar la seguridad y eficacia del antiangiogénico versus observación en ojos con RDNP grave, AV $\geq 20 / 25$ sin EMD y sin historia de tratamiento previo, como herramienta preventiva de aparición de EMD o RDP.

\section{Protocolo AA}

Evaluará lesiones de RD periféricas por imágenes de campo ultraamplio (UCA) y su asociación con el empeoramiento de la gravedad de la RD. El objetivo es saber si el estudio de imágenes de la periferia con fotografías de UCA, otorga posibilidad de evaluar la RD y predecir su tasa de empeoramiento con el tiempo, comparada con fotografías estándares de los siete campos del ETDRS. Se incluirán ojos con RDNP, sin edema macular y sin antecedente de tratamiento. Se les realizarán controles anuales durante 4 años para evaluar el riesgo relativo de empeoramiento de dos o más niveles de gravedad en grupos con y sin lesiones periféricas mediante imágenes de UCA (Optos).

\section{Protocolo $A B$}

Comprarará el tratamiento con antiangiogénico intravítreo (aflibercept) contra la vitrectomía temprana para el tratamiento de la hemorragia vítrea por RDP. El objetivo principal es comparar la AV a los 6 meses después de cada una de las intervenciones. En el grupo de antiangiogénico se aplicarán al menos 2 dosis mensuales al inicio y, posteriormente por razón necesaria, se podrá realizar en ellos vitrectomía, hasta 4 meses después, en caso de no aclaramiento de la hemorragia, y PFC en caso de no ser suficiente con el 
antiangiogénico para el tratamiento de la enfermedad. En el grupo de vitrectomía, esta se realizará con PFC intraoperatoria en las primeras 2 semanas después de la aleatorización. Se podrán aplicar inyecciones de antiangiogénico en caso de presentar recurrencia de la hemorragia.

\section{Protocolo AC}

Consistirá en la administración de aflibercept intravítreo vs. bevacizumab con aflibercept diferido para el del EMD con involucración central. El objetivo es comparar la eficacia en cuanto a cambio de la AV con cada una de las conductas terapéuticas en pacientes con dicho diagnóstico. Los pacientes del grupo de bevacizumab serán candidatos a cambio de fármaco hacia aflibercept a partir de las 12, 16, 20 y 24 semanas en caso de cumplir los criterios establecidos.

\section{Protocolo AD (estudio PROMINENT)}

Tiene como objetivo evaluar si el tratamiento con pemfibrato $(0.2 \mathrm{mg} / 12 \mathrm{~h}$ vía oral) comparado con placebo reduce la tasa de empeoramiento de la $\mathrm{RD}$ en pacientes diabéticos tipo 2 con RDNP.

\section{Protocolo GEN}

El estudio tiene como objetivo crear un banco de material genético e información sobre el fenotipo clínico para la comunidad científica, con la justificación de que en un futuro se nos pueda brindar la suficiente información que permita evaluar la susceptibilidad o resistencia genética en temas referentes a RD. Así mismo, se pretende determinar variantes que puedan impactar sobre biomarcadores clave en el desarrollo del EMD y neovascularización.

\section{Conclusión}

Durante los últimos años, la red del DRCR nos han proveído de resultados clínicos a preguntas de investigación importantes referentes al tratamiento del EMD $y$, recientemente, al de la RD.

Se ha dado respuesta a cuestiones de modalidades de tratamiento e innovaciones tecnológicas al respecto, ya sea con asociación directa o de carácter secundario, que de otra manera no habrían sido contestadas por la industria o por otras entidades.

Los antiangiogénicos se han aceptado globalmente como el tratamiento de primera línea en el manejo del
EM, y han desplazado el láser focal a un adyuvante en casos seleccionados y han dejado en desuso la aplicación de láser macular en parrilla. Lo que actualmente está aún en discusión es el manejo de la RDP. Es ya bien sabida la no inferioridad; sin embargo, aún es controvertida su elección como terapia de primera línea. Asimismo, los medicamentos esteroideos han demostrado cierta utilidad, aunque actualmente continúan siendo un tratamiento de segunda línea para el EMD.

Se debe destacar que las conclusiones que nos brinda la red son resultado de una colaboración entre un grupo de investigadores clínicos que participan en centros académicos, así como en prácticas privadas, lo que, al menos en teoría, conlleva el hecho de poder extrapolar correctamente los resultados a la práctica clínica diaria. No obstante, sugerimos que los resultados deben ser considerados con extrema cautela, y mantener en el primer plano las características particulares de nuestro paciente, nuestro entorno y el objetivo principal, que es el tratar de obtener el mejor beneficio para el paciente por medio de la terapia seleccionada.

\section{Financiamiento}

El presente trabajo fue financiado por el patronato de la Fundación Hospital Nuestra Señora de la Luz, Institución de Asistencia Privada. La organización de financiación no tuvo ningún rol en el diseño o conducta de la presente investigación.

\section{Conflicto de intereses}

No existe ningún a relación de conflicto de intereses por parte de ningún autor.

\section{Responsabilidades éticas}

El presente trabajo ha sido evaluado y autorizado con el número AR-Ret-0015.

Protección de personas y animales. Los autores declaran que para esta investigación no se han realizado experimentos en seres humanos ni en animales.

Confidencialidad de los datos. Los autores declaran que en este artículo no aparecen datos de pacientes.

Derecho a la privacidad y consentimiento informado. Los autores declaran que en este artículo no aparecen datos de pacientes. 


\section{Bibliografía}

1. Diabetic Retinopathy Clinical Research Network. Comparison of the modified Early Treatment Diabetic Retinopathy Study and mild macular grid laser photocoagulation strategies for diabetic macular edema. Arch Ophthalmol. 2007;125(4):469-8.

2. Diabetic Retinopathy Clinical Research Network. The Relationship between OCT-measured Central Retinal Thickness and Visual Acuity in Diabetic Macular Edema. Ophthalmology. 2007;114(3):525-36.

3. Diabetic Retinopathy Clinical Research Network. Comparison of Time-Domain OCT and Fundus Photographic Assessments of Retinal Thickening in Eyes with Diabetic Macular Edema. Invest Ophthalmol Vis Sci. 2008;49(5):1745-52.

4. Diabetic Retinopathy Clinical Research Network. Association of the extent of diabetic macular edema as assessed by optical coherence tomography with visual acuity and retina outcome variables. Retina. 2009; 29:300-5.

5. Diabetic Retinopathy Clinical Research Network. Association of Fluorescein Angiographic Features with Visual Acuity and with Optical Coherence Tomographic and Stereoscopic Color Fundus Photographic Features of Diabetic Macular Edema in a Randomized Clinical Trial. Retina. 2010;30(10):1627-37.

6. Diabetic Retinopathy Clinical Research Network. A Randomized Trial Comparing Intravitreal Triamcinolone Acetonide and Focal/Grid Photocoagulation for Diabetic Macular Edema. Ophthalmology. 2008;115(9) 1447-9.

7. Diabetic Retinopathy Clinical Research Network. Three-year Follow Up of a Randomized Trial Comparing Focal/Grid Photocoagulation and Intravitreal Triamcinolone for Diabetic Macular Edema. Arch Ophthalmol. 2009;127(3):245-51

8. Diabetic Retinopathy Clinical Research Network. Diurnal Variation in Retinal Thickening Measurement by OCT in Center-involved Diabetic Macular Edema. Arch Ophthalmol. 2006;124(12):1701-7.

9. Diabetic Retinopathy Clinical Research Network. Vitrectomy Outcomes in Eyes with Diabetic Macular Edema and Vitreomacular Traction. Ophthalmology. 2010;117(6):1087-93.e3.

10. Diabetic Retinopathy Clinical Research Network. Factors associated with visual acuity outcomes after vitrectomy for diabetic macular edema. Retina. 2010:30:1488-95.

11. Diabetic Retinopathy Clinical Research Network. A Randomized Trial of Peribulbar Triamcinolone Acetonide with and without Focal Photocoagulation for Mild Diabetic Macular Edema: A Pilot Study. Ophthalmology. 2007:114(6):1190-6.

12. Diabetic Retinopathy Clinical Research Network. An Observational Study of the Development of Diabetic Macular Edema Following Panretina (Scatter) Photocoagulation (PRP) Given in 1 or 4 Sittings. Arch Ophthalmol. 2009;127(2):132-40.

13. Bressler NM, Edwards AR, Antoszyk AN, Beck RW, Browning DJ, Ciardella AP, et al. Retinal Thickness on Stratus Optical Coherence Tomography in People with Diabetes and Minimal or No Diabetic Retinopathy. Am J Ophthalmol. 2008;145:894-901.

14. Diabetic Retinopathy Clinical Research Network. Observational study of subclinical diabetic macular edema. Eye. 2012;26:833-40.

15. Diabetic Retinopathy Clinical Research Network. A Phase 2 Randomized Clinical Trial of Intravitreal Bevacizumab for Diabetic Macular Edema. Ophthalmology. 2007;114(10):1860-7.

16. The Diabetic Retinopathy Clinical Research Network. Randomized Trial Evaluating Ranibizumab Plus Prompt or Deferred Laser or Triamcinolone Plus Prompt Laser for Diabetic Macular Edema. Ophthalmology. 2010;117(6):1064-77.e35

17. The Diabetic Retinopathy Clinical Research Network. Expanded 2-Year Follow-up of Ranibizumab Plus Prompt or Deferred Laser or TriamcinoIone Plus Prompt Laser for Diabetic Macular Edema. Ophthalmology. 2011;118(4):609-14.

18. Diabetic Retinopathy Clinical Research Network. Intravitreal Ranibizumab for Diabetic Macular Edema with Prompt vs Deferred Laser Treatment: 3-year Randomized Trial Results. Ophthalmology. 2012;119(11): 2312-8.

19. Diabetic Retinopathy Clinical Research Network. Intravitreal Ranibizumab for Diabetic Macular Edema with Prompt versus Deferred Laser Treatment: 5-Year Randomized Trial Results. Ophthalmology. 2015;122(2): 375-81.

20. Diabetic Retinopathy Clinical Research Network. Randomized Trial Evaluating Short-Term Effects of Intravitreal Ranibizumab or Triamcinolone Acetonide on Macular Edema Following Focal/Grid Laser for Diabetic Macular Edema in Eyes Also Receiving Panretinal Photocoagulation. Retina. 2011;31(6):1009-27.
21. Diabetic Retinopathy Clinical Research Network. The Course of Response to Focal/Grid Photocoagulation for Diabetic Macular Edema. Retina. 2009;29(10):1436-43.

22. Diabetic Retinopathy Clinical Research Network. Evaluation of Visual Acuity Measurements After Autorefraction vs Manual Refraction in Eyes With and Without Diabetic Macular Edema. Arch Ophthalmol. 2012; 130(4):470-9.

23. Dagogo-Jack S, Funnell MM, Davidson J. Barriers to achieving optimal glycemic control in a multi-ethnic society: a US focus. Curr Diabetes Rev. 2006;2(3):285-93.

24. Davis MD, Fisher MR, Gangnon RE, Barton F, Aiello LM, Chew EY, et al. Risk factors for high-risk proliferative diabetic retinopathy and severe visual loss: ETDRS report \#18. Invest Ophthalmol Vis Sci. 1998;39:233-52.

25. Stratton IM, Kohner EM, Aldington SJ, Turner RC, Holman RR, Manley SE, et al. UKPDS 50: risk factors for incidence and progression of retinopathy in Type II diabetes over 6 years from diagnosis. Diabetologia. 2001;44(2):156-63.

26. The Diabetes Control and Complication Trial Research Group. The effect of intensive treatment of diabetes on the development and progression of long-term complication in insulin-dependent diabetes mellitus. N Engl J Med. 1993:329:977-86.

27. Diabetic Retinopathy Clinical Research Network Writing Committee. Cluster Randomized Trial Assessing the Effect on Diabetes Control of Personalized Diabetes Complication Risk Assessment during Ophthalmology Exams. JAMA Ophthalmol. 2015;133(8):888-96.

28. Diabetic Retinopathy Clincical Research Network. Randomized clinical trial evaluating intravitreal ranibizumab or saline for vitreous hemorrhage from proliferative diabetic retinopathy. JAMA Ophthalmol. 2013; 131(3):283-93.

29. Diabetic Retinopathy Clinical Research Network. Evaluation of Results 1 Year Following Short-term Use of Ranibizumab for Vitreous Hemorrhage Due to Proliferative Diabetic Retinopathy, JAMA Ophthalmol. 2014; 132(7):889-90.

30. Diabetic Retinopathy Clinical Research Network. Retinal Thickness in People with Diabetes and Minimal or No Diabetic Retinopathy: Heidelberg Spectralis Optical Coherence Tomography. Invest Ophthalmol Vis Sci. 2012:53:8154-61.

31. The Diabetic Retinopathy Clinical Research Network. Reproducibility of Spectral Domain Optical Coherence Tomography Retinal Thickness Measurements and Conversion to Equivalent Time Domain Metrics in Diabetic Macular Edema. JAMA Ophthalmol. 2014:132(9):1113-22.

32. Bressler SB, Edwards AR, Andreoli AM, Edwards PA, Glassman AR, Jaffe GJ, et al. Reproducibility of Optovue RTVue optical coherence tomography retinal thickness measurements and conversion to equivalent Zeiss stratus metrics in diabetic macular edema. Tran Vis Sci Tech. 2015;4(1):5.

33. Diabetic Retinopathy Clinical Research Network Authors/Writing Committee. Pilot Study of Individuals With Diabetic Macular Edema Undergoing Cataract Surgery. JAMA Ophthalmol. 2014;132(2):224-6.

34. Diabetic Retinopathy Clinical Research Network Authors/Writing Committee, Baker CW, Almukhtar T, et al. Macular Edema After Cataract Surgery in Eyes Without Preoperative Central-Involved Diabetic Macular Edema. JAMA Ophthalmol. 2013;131(7):870-9.

35. Friedman SM, Almukhtar TH, Baker CW, Glassman AR, Elman MJ, Bressler NM; Diabetic Retinopathy Clinical Research Network. Topical nepafenec in eyes with non-central diabetic macular edema. Retina. $2015 ; 35(5): 944-56$

36. Diabetic Retinopathy Clinical Research Network. Panretinal Photocoagulation vs Intravitreous Ranibizumab for Proliferative Diabetic Retinopathy: A Randomized Clinical Trial, JAMA Ophthalmol. 2015;314(20):2137-46.

37. Diabetic Retinopathy Clinical Research Network. Cost-effectiveness of Intravitreous Ranibizumab Compared With Panretinal Photocoagulation for Proliferative Diabetic Retinopathy Secondary Analysis From a Diabetic Retinopathy Clinical Research Network Randomized Clinical Trial. JAMA Ophthalmol 2017;135(6):576-84.

38. Diabetic Retinopathy Clinical Research Network. Five-Year Outcomes of Panretinal Photocoagulation vs Introus Ranibizumab for Proliferative Diabetic Retinopathy: A Randomized Clinical Trial. JAMA Ophthalmol. 2018;136(10):1138-48

39. Diabetic Retinopathy Clinical Research Network; Wells JA, Glassman AR, Ayala AR, Jampol LM, Aiello LP, Antoszyk AN, et al. Aflibercept, bevaczumab, or ranibizumab for diabetic macular edema. $\mathrm{N}$ Engl $\mathrm{J}$ Med. 2015;372:1193-203.

40. Diabetic Retinopathy Clinical Research Network Aflibercept. Bevacizumab, or Ranibizumab for Diabetic Macular Edema Two-Year Results from a Comparative Effectiveness Randomized Clinical Trial. Ophthalmology. 2016:123(6):1351-9.

41. Diabetic Retinopathy Clinical Research Network. Effect of Adding Dexamethasone to Continued Ranibizumab, Treatment in Patients With Persistent Diabetic Macular Edema. JAMA Ophthalmol. 2018;136(1):29-38. 